\title{
HIPAA violations among nursing students: Teachable moment or terminal mistake-A case study
}

\author{
Annette A. Cannon ${ }^{*}$ Hollie Caldwell \\ School of Nursing, Platt College, Aurora, Colorado, United States
}

Received: May 18, 2016

Accepted: July 11, 2016

Online Published: July 20, 2016

DOI: $10.5430 /$ jnep.v6n12p41

URL: http://dx.doi.org/10.5430/jnep.v6n12p41

\begin{abstract}
A case study involving one nursing education program's experience with a Health Insurance Portability and Accountability Act (HIPAA) violation is used to illustrate how one nursing college dealt with a student's HIPAA violation. HIPAA violations committed by students within healthcare education programs are under-studied and under-discussed. HIPAA violations made by nursing students are complex because they occur during the learning process and can involve a variety of variables including clinical facility responsibilities, dual roles of nursing student and clinical facility employee, the appropriateness of patient assignments, the role and accountability of clinical facility nurse educators, nursing unit culture, and staff nurse role modeling. Findings reveal that nursing students are at a higher vulnerability than previously known, for HIPAA violations. This case study serves as an example of how one nursing program decided to use a HIPAA violation by a senior nursing student, and use it as a teachable moment, rather than a terminal mistake.
\end{abstract}

Key Words: Health Insurance Portability and Accountability Act, Nursing students, Nursing education, Violations, Case study

\section{INTRODUCTION}

A student was enrolled in a 180-hour senior practicum course within a traditional pre-licensure baccalaureate nursing program offered in the mid-west. This student was assigned to the acute care facility in which she was also employed as a CNA. Consistent with the policy of the acute care agency, the student was assigned to night shifts on the same unit on which she was employed. As part of the on-boarding process for this practicum, the student signed an acknowledgment stating she had received and understood the facility's expectations relating to the Health Insurance Portability and Accountability Act (HIPAA).

\section{The case}

On the first day of clinical, the student was given a verbal assignment to select and present a patient and family for which the student had provided care and discuss the impact of fam- ily dynamics and/or family support on the nursing student's ability to care for the patient. The student was instructed to be prepared to present this individual case study in two weeks to other senior practicum students from a variety of nursing education programs and representatives from the facility's education department. The nursing education program assigned a senior practicum faculty oversight to the student. This full time nursing faculty was responsible for evaluating student achievement of curricular objectives/outcomes related to nursing knowledge and practice as well as planning, monitoring and evaluating the instruction provided by the facility preceptors.

The afternoon of the case presentation, the Director of Clinical Education from facility telephoned the clinical placement coordinator (CPC) for the nursing education program to inform her that the student had been terminated from the senior practicum experience effective that date, due to a HIPAA

*Correspondence: Annette A. Cannon; Email: annette.cannon @ plattcolorado.edu; Address: 3100 S. Parker Rd., Suite 200, Aurora, United States. 
violation which occurred during "an informal discussion of their patient to the student group". She went on to state the student was "unprofessional in her approach to the discussion of a family situation, shared HIPAA information concerning the family situation, discussed a case in which the hospital was currently reviewing, and shared patient information after having received a directive in her role as employee of the hospital not to discuss this patient case".

At the nursing program, the CPC immediately notified the nursing education program's Senior Practicum Team, composed of the student's senior practicum oversight faculty, course faculty, and the Associate Dean. The student was contacted and asked not to return to clinical and to wait until the Team could gather additional information. The student informed the Team that she had also been contacted by the facility's Human Resources department and was told she was not to return to work for shifts as a CNA until further notice.

Upon discussion with the student, the student did not see feel that she had done anything wrong and was surprised by the accusations. It was determined that the student had cared for the patient both as a student nurse and as an employee. The student maintained that her preceptor, who was not her primary preceptor, but a staff nurse who had filled in on during the preceptor's vacation, suggested that this particular patient would make an excellent student case study due to complicated family dynamics, complex medical history, prolonged length of stay, and high profile nature of the case. They also agreed that working night shift had provided the student very little interaction with patient's families leaving very few case possibilities from which to choose.

The student did admit that both she and the substitute preceptor were aware that this patient's family was currently in review with the hospital and that they had received directives as employees of the facility not to discuss issues related to this patient. The student stated she shared the parents' general occupations as being lawyers and prominent public figures in the community, because she felt this information was relevant to how she provided care to the patient. She states she did not state the names of the parents or patient, the medical record or room number, the names of the companies in which the parents worked, or any other specific patient identifiers.

In her discussion with her senior practicum oversight faculty, the student went on to share that she had presented the case in a way similar to what she did as a CNA when employed at the facility during report and in a manner similar to the licensed nurses on the unit. She shared that is was the "cultural norm" on this unit for the nursing to freely and openly discuss the same content she felt she had given during her case presentation. The student stated she was confused by why the standards for the verbal presentation required as an assignment for the senior practicum would be different than how patients were discussed in agency hand-off reports among the CNA's and professional nursing staff.

The Associate Dean sought additional clarification about the assignment and the students' case presentation directly from the Director of Clinical Education. The Director was present at the presentation and stated that during the case presentation the student had disclosed the occupations of the parents and that the hospital was currently in litigation with the family. She also described being "offended" by the flippant manner in which the material was presented by the student. She added that the student had made comments regarding the quality of care provided by the nurses at this facility and had "blamed the nurses for the patient's complications and prolonged length of stay". The director also stated that she was "so paralyzed with shock" during the student's case presentation, she made no attempt to stop or correct the student during the verbal presentation. The director confirmed that the presentations were not video recorded and no written work was turned in as part of the assignment.

After all of the approximately 25 case presentations were complete, the director stated she privately informed the student that she could not return for the practicum due to violating HIPAA. She also sent an email to the student, involved faculty, and the Dean of the nursing education program stating the student was dismissed from the clinical setting for "an unprofessional approach to discussion of a family situation; sharing of HIPAA information concerning a family situation; discussing a case in which the clinical agency was currently under litigation; and sharing patient information after receipt of a directive while employed as a CNA that information related to this patient and family was not to be shared in any situation." Because the nursing education director knew the student was also employed as a CNA at the facility, she stated she had also notified the facility's human resources department about the HIPAA violation.

After review of the policy of the nursing education program, it was determined by the Team that the behaviors described by the Director of Clinical Education, at the very least, constituted a violation of the professional behavior policy and was unacceptable for a nursing student enrolled in a senior practicum course. The policy allowed discipline including verbal or written reprimand, temporary suspension, or dismissal from the nursing education program. The Associate Dean and the student's practicum oversight faculty embarked on a literature search to explore instances in the literature in which issues HIPAA violations by nursing students were 
discussed in the literature for further guidance before determining whether this was a teachable moment or a terminal mistake.

\section{BACKGROUND}

Although the law has been in effect for the last eighteen years, it continues to be updated to accommodate changes within the healthcare system. This law has had a significant impact on the health care industry including the need for numerous changes in the way we communicate with our patients, their families, and with each other. ${ }^{[1]}$ As nurses, we are reminded to keep patient information private. The United States Department of Health and Human Services (USDHHS) conducts the updates on this law and the Office of Civil Rights (OCR) enforces the law. ${ }^{[2]}$ For example, some recent updates in the law have been due to the Patient Protection and Affordable Care Act of 2010 (PPACA), the Health Information Technology for Economic and Clinical Health (HITECH) of 2009 and the related Omnibus HIPAA Rule (2013). These updates occur to help the healthcare system run efficiently and effectively, to strengthen the enforcement of the rules through both criminal and civil action, and to strengthen, and to expand existing provisions in light of the increased use of technology to disseminate personal health information.

Consumers of health care expect that their information will be held sacred and kept private by the healthcare provider caring for them. The law has provided rights and protections to patients, not only with regard to their health information, but how it is shared with others under certain circumstances. These rights, protections and circumstances are detailed within the rules, regulations and guidelines of the law. The HIPAA was enacted into law in 1996 and impacted the health- care industry by changing how we communicate with our patients, their families, and other members of the healthcare team and by providing rights to patients and safeguarding healthcare employees. ${ }^{[1]}$ Seven years later, the Department of Health and Human Services (HHS) developed and implemented the Standards for Privacy of Individually Identifiable Health Information also known as the Privacy Rule to regulate privacy and confidentiality of medical records.

Terms sometimes used interchangeably that are important to understand include individually identifiable health information, health information, and protected health information (PHI) (see Table 1). "Individually identifiable health information is a subcategory of health information, including demographic information collected from an individual, and: (1) is created or received by a health care provider, health plan, employer, or health care clearinghouse; and (2) relates to the past, present, or future physical or mental health or condition of an individual; the provision of health care to an individual; or the past, present, or future payment for the provision of health care to an individual; and (3) that identifies the individual"; or (4) information for which it could be reasonably believed could be used to identify the individual. ${ }^{[3]}$ Health information is more broad and includes "any information, whether oral or recorded in any form or medium, that (1) is created or received by a health care provider, health plan, public health authority, employer, life insurer, school or university, or health care clearinghouse; and (2) relates to the past, present, or future physical or mental health or condition of any individual, the provision of health care to an individual, or the past, present, or future payment for the provision of health care to an individual". ${ }^{[2]} \mathrm{PHI}$ includes individually identifiable health information (see Table 2).

Table 1. Definitions of Important HIPAA Privacy Rule Terms ${ }^{[4]}$

\begin{tabular}{|c|c|c|}
\hline Health Information & Individually Identifiable Health Information & Protected Health Intor \\
\hline $\begin{array}{l}\text { Any information, whether oral or } \\
\text { recorded in any form or medium, } \\
\text { that- } \\
\text { (A) is created or received by a health } \\
\text { care provider, health plan, public } \\
\text { health authority, employer, life } \\
\text { insurer, school or university, or } \\
\text { health care clearinghouse; and } \\
\text { (B) relates to the past, present, or } \\
\text { future physical or mental health or } \\
\text { condition of any individual, the } \\
\text { provision of health care to an } \\
\text { individual, or the past, present, or } \\
\text { future payment for the provision of } \\
\text { health care to an individual. }\end{array}$ & $\begin{array}{l}\text { This is a subset of health information, including } \\
\text { demographic information collected from an } \\
\text { individual, and: } \\
\text { (1) Is created or received by a health care provider, } \\
\text { health plan, employer, or health care clearinghouse; } \\
\text { and } \\
\text { (2) Relates to the past, present, or future physical or } \\
\text { mental health or condition of an individual; the } \\
\text { provision of health care to an individual; or the past, } \\
\text { present, or future payment for the provision of health } \\
\text { care to an individual; and } \\
\text { (i) That identifies the individual; or } \\
\text { (ii) With respect to which there is a reasonable basis } \\
\text { to believe the information can be used to identify the } \\
\text { individual. }\end{array}$ & $\begin{array}{l}\text { Individually identifiable health information } \\
\text { that is: } \\
\text { (i) Transmitted by electronic media; } \\
\text { (ii) Maintained in electronic media; or } \\
\text { (iii) Transmitted or maintained in any other } \\
\text { form or medium. } \\
\text { There are two exceptions: } \\
\text { Individually identifiable health information } \\
\text { in records covered by Family Educational } \\
\text { and Privacy Rights (FERPA); and } \\
\text { Individually identifiable health information } \\
\text { in employment records held by a covered } \\
\text { entity in its role as employer. }\end{array}$ \\
\hline
\end{tabular}


The HIPAA Privacy Rule stipulates that privacy can be maintained by two methods of de-identification. The Standard method is "Health information [defined above] that does not identify an individual and with respect to which there is no reasonable basis to believe that the information can be used to identify an individual is not individually identifiable health information". ${ }^{2]}$ Implementation specifications for de-identification require statistically and scientifically sound methods to be used to ensure there is very little chance of identifying an individual by the information provided. The 18 identifiers that must be removed extend beyond just the patient or individual and include the protected health information of identifiers of relatives, employers, or other household members (see Table 2).

Table 2. 18 Protected Health Information Identifiers Must Be Removed to Meet HIPAA Rule De-Identification Requirements ${ }^{[4]}$

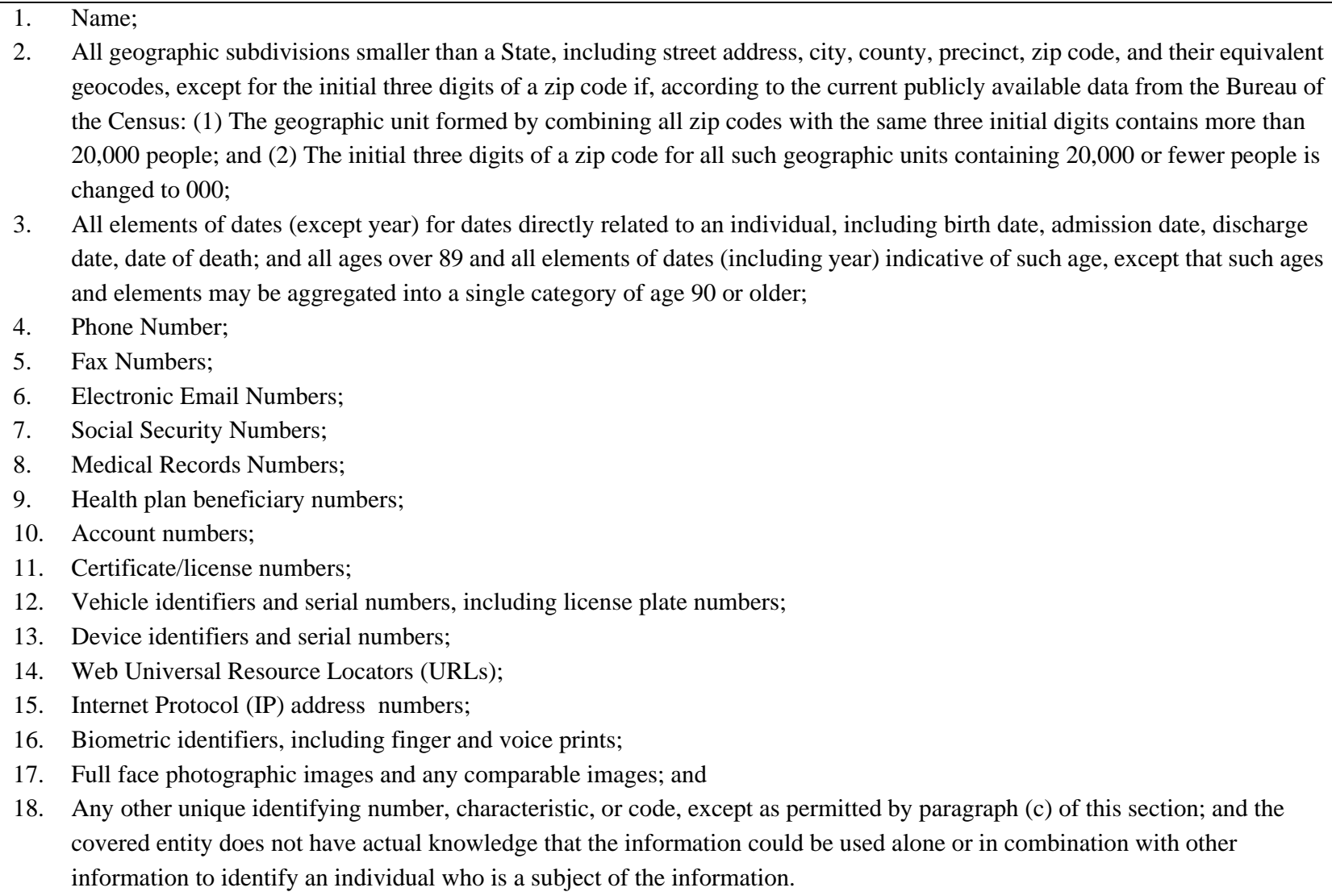

In healthcare, HIPAA violations can result in escalating civil and criminal penalties, which can be imposed by State Attorneys General, the Department of Justice (DOJ) or, if the DOJ declines, the Office of Civil Rights in the Department of Health and Human Services (see Table 3). ${ }^{[2]}$ The issues of reasonable cause and willful neglect are important considerations in HIPAA Rule enforcement. Reasonable cause means the "means an act or omission in which a covered entity or business associate knew, or by exercising reasonable diligence would have known, that the act or omission violated an administrative simplification provision, but in which the covered entity or business associate did not act with willful neglect". ${ }^{[2]}$ Willful neglect, on the other hand, means "conscious, intentional failure or reckless indifference to the obligation to comply with the administrative simplifi- cation provision violated". ${ }^{[4]}$ Investigations of allegations of HIPAA violations, the extent and nature of the violation as well as the extent and nature of any harm, either physical or financial, caused by the violation. ${ }^{[2]}$ There are four tiers of penalties for HIPAA rule enforcement (see Table 3).

Despite the fact that nursing students are rarely involved in the electronic transmission of PHI, nursing students are held to the same standard as licensed nurses who frequently transmit transactions containing PHI. Ultimately, nursing students must comply with the general principle of the Privacy Rule and as a covered entity "may not use or disclose protected health information, except either: (1) as the Privacy Rule permits or requires; or (2) as the individual who is the subject of the information (or the individual's personal representative) authorizes in writing". ${ }^{[5]}$

ISSN 1925-4040 E-ISSN 1925-4059 
Table 3. Four Tiers of Penalty for HIPAA Rule Enforcement

\begin{tabular}{lll}
\hline Violation Category & Each Violation & All such violations of an identical provision in a calendar year \\
\hline (A) Did Not Know & $\$ 100-\$ 50,000$ & $\$ 1,500,000$ \\
(B) Reasonable Cause & $\$ 1,000-\$ 50,000$ & $\$ 1,500,000$ \\
(C) (i) Willful Neglect-Corrected & $\$ 10,000-\$ 50,000$ & $\$ 1,500,000$ \\
(C) (ii) Willful Neglect-Not Corrected & $\$ 50,000$ & $\$ 1,500,000$ \\
\hline
\end{tabular}

Educators have an inherent obligation to ensure nursing students are educated in their ethical and legal responsibilities to protect and maintain the privacy of individuals within their care. Nursing students participate in a variety of clinical learning experiences as part of the educational process and provide medical or health services qualifying them as a covered entity subject to compliance to the Privacy Rule. ${ }^{[5]}$ A variety of charts exist to help with determination of covered entity status (see Figure 1). These can be found at http://www.cms.gov

\section{Is a person, business, or agency a covered health care provider?}

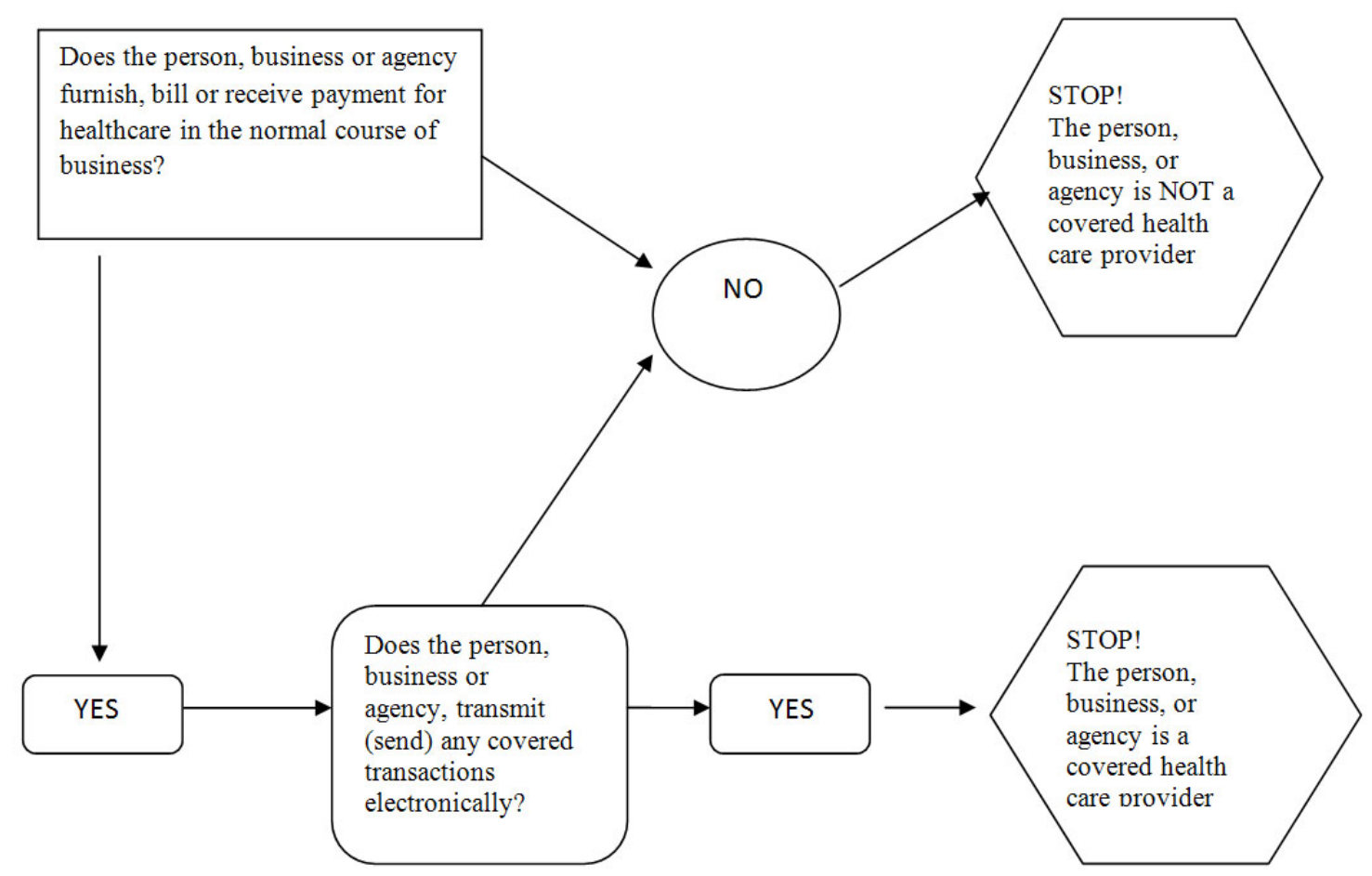

Figure 1. HIPAA Covered Entity Chart from Centers for Medicare \& Medicaid Services (CMS)

In nursing education, a HIPAA violation made by a nursing student could result in a variety of disciplinary actions including termination, but is rarely discussed in nursing literature. Terminating a student in the last quarter of the baccalaureate nursing program is a serious matter for all involved. Such a termination can result in the dismissed student experiencing difficulty with credit transfer to another nursing program and a large monetary investment in an education for which they cannot become licensed or employed. Alternatively, nursing education programs have a duty to ensure nursing graduates are competent to protect the safety and confidentiality of all patients and their families under their care.

With HIPAA Rule enforcement standards and the unique details of this case in mind, the Associate Dean and the student's senior practicum oversight faculty embarked on an investigation to determine HIPAA Rule violation category (A, B, C [i]), or C [ii]) and to explore the roles of the nursing education program, the student, and the clinical agency in this HIPAA violation (see Table 1). The Team agreed that more information was needed before determining whether 
this was a teachable moment or a terminal mistake.

\section{The Role of THE NURSING EDUCATION PROGRAM}

College clinical performance expectations are covered during nursing orientation and again throughout the nursing curriculum as student's progress in their clinical education. They are also available to students on the college website, located under college policies. This information states any "nursing student demonstrating a pattern of unsafe performance, lack of accountability, or inconsistency in performance in the clinical area, may be immediately terminated from the clinical area by the clinical faculty, even if there are days remaining in the clinical rotation". It also states that students can be terminated from the clinical education rotation for concerns voiced by the clinical agency. It goes on to state if clinical agency or facility requests that a student NOT return to their facility (to complete a clinical rotation the student is currently taking or for a future clinical) due to behavioral issues, substance abuse/drugs, etc., the nursing program has the right to terminate a clinical experience and/or assign a failing grade. Such a failure can impact future completion of coursework and continuation in the nursing program. The college's Clinical Placement Policy emphasizes the professional behaviors expected in clinical areas and requires that students to "maintain a professional demeanor at all times in the clinical area", per the 2015 Platt College Policy. It adds that students are expected to "communicate professionally, positively, and respectfully with patients/clients, patient/client families, faculty, other students, agency staff, and community professionals and adhere to the HIPAA guidelines at all times".

It is known that nurses rank the highest among the public in trust and are rated the most honest and ethical among professions. ${ }^{[6]}$ Very often it is the nurse in which the patient confides and nurses use judgment when deciding what is appropriate to share and what is on a need to know basis to provide optimal care for the patient. "Sharing information between members of the treating team, or between different treating practitioners, is a common and necessary practice in the delivery of healthcare". ${ }^{[7]}$ This sharing of information occurs during handoff reports, patient rounds, and informal patient case presentations by all members of the healthcare team. The importance of protection of confidential information concerning patients and their families is emphasized in nursing education because "... the exposure of someone's PHI can ruin someone's livelihood, affect their family relationships, and change the course of their life". ${ }^{[8]}$ Violations of patient confidentiality can also result in the nurse being disciplined or losing their job, or their license. ${ }^{[5]}$ Despite having received education on HIPAA and their ethical obligation to protect and maintain patient privacy, nursing students may still be vulnerable to making mistakes. Nurse educators and preceptors must be knowledgeable about HIPAA and aware of circumstances or assignments that may increase the likelihood of a HIPAA violation by a nursing student.

\section{HIPAA AND THE NURSING STUDENT}

As a result of the senior practicum, the oversight faculty and the Associate Dean conducted a review of a sample of four documents from the major health systems (two teaching and research missions, one for-profit, and one not-for-profit institutions) in the local area that students in the nursing program are required to sign. They also reviewed the instructional material on HIPAA provided to students as part of the new nursing student orientation in the nursing program. This material was developed in 2008 by a coalition of nurse educators and clinical educators from clinical facilities and is reviewed and updated by a subcommittee of this organization every two years. This 55 slide training describes the HIPAA Privacy rules and regulations; identifies patients' rights and the student nurse's role in protecting them; discusses the student nurse's responsibilities under HIPAA, and provides related policies and procedures; and explains the penalties for non-compliance with HIPAA. Definitions were provided for HIPAA; Patient Confidentiality; PHI; and Transaction. Protected privacy elements are listed and nine scenarios and answers relevant to the functions of a student nurse are also provided. All four forms covered confidentiality and security requirements related to HIPAA. All forms used the term "confidential Information", however, the term "protected health information" was not used or defined.

The student had been oriented to HIPAA expectations at multiple points across the curriculum in the nursing program and at the facility to which she was currently assigned. Her student file contained several signed acknowledgments attesting to her receipt and understanding of about the student's role in protecting and maintaining patient privacy and adherence to the HIPAA regulation. Based on the results of this review, the Team agreed that the nursing education program had provided adequate education on HIPAA and that the violation was not related to a knowledge deficit.

\section{EXAMINING THE ROLE OF THE STUDENT}

\subsection{Previous performance}

The Team considered that this student had had one other disciplinary incident not related to patient confidentiality or safety early in her academic career, but otherwise attended courses regularly and produced satisfactory work products in class, lab, and practicum. Most members of the Team 
had had the student in classes and agreed that they could see where the student's verbal approach could be perceived as "flippant", but had not experienced the student being disrespectful when speaking about a patient or patient care.

\subsection{Reasonable cause or willful neglect?}

In discussing the incident with the student, the Team decided she was not recklessly indifferent to her obligation to comply with HIPAA nor did the Team believe she consciously or intentionally violated this Rule.

\subsection{Student's dual role of employee and student nurse}

In her discussion with her senior practicum oversight faculty, the student went on to share that she had presented the case in a manner similar to what she had done in her role as a CNA at the facility and in a manner similar to what she had witnessed the licensed nurses provide on the unit. She shared that is was the "cultural norm" on this unit for the nursing to freely and openly discuss the same content she felt she had given during her case presentation. The student was confused by why the standards for the verbal presentation required as an assignment for the senior practicum would be different than how patients were discussed in agency hand-off reports among the CNA's and professional nursing staff. It was apparent in discussions and a written statement prepared by the student that she felt she had replicated the same type of hand-off report during the case presentation that she had seen role-modeled by licensed nursing working at the facility.

\section{Determining THE ROLE OF THE CLINI- CAL AGENCY}

The student had been provided a Student Manual by the clinical facility which stated that HIPPA violations could result in immediate dismissal from the clinical facility. The Clinical Education Director confirmed that the student's case presentation was not video recorded and no written work was turned in as part of the assignment. Additionally, she stated there was no approval process for the case selected by the students for this assignment. Furthermore, the student's senior practicum oversight faculty had not been provided with the assignment guidelines nor had she been invited to attend the case presentations. Another concern for the Team was the failure of the Director of Education to immediately intervene in order to prevent or stop a potential HIPAA violation.

\section{DETERMINING THE SUPPORT NEEDED BY STUDENT}

The student was provided support from the practicum oversight faculty member throughout this event. Frequent meetings were held to allow the student to ask questions and gain

Published by Sciedu Press information. The practicum oversight faculty reviewed the situation with the student and offered feedback, guidance and reassurance. Frequent availability by the Dean, Clinical Coordinator and practicum oversight faculty for discussions and review, continued until the situation was resolved. The student was also provided with resources from Student Services if needed.

\section{Determining THE CONSEQUENCES OF THE HIPAA VIOLATION}

After review of the policy of the nursing education program, it was determined by the Team that the student behaviors described by the Director of Clinical Education, at the very least, constituted a violation of the professional behavior policy and was unacceptable for a nursing student enrolled in a senior practicum course. The policy allowed discipline including verbal or written reprimand, temporary suspension, or dismissal from the nursing education program.

There was an obvious gap in communication and oversight between the clinical agency and the nursing education program regarding the case presentation assignment. Other factors seemed to have been larger contributors to the HIPAA violation. Of particular concern for the Team was the double jeopardy situation created by the student's dual roles of employee and senior practicum student at the facility. A mistake made while fulfilling the role of senior practicum nursing student was now impacting the student's ability to return to work as a CNA. Based on all of the information available, the Team decided on the following actions: The student was determined to be in violation of the college's Professional Behavior Policy resulting in suspension from the nursing education program for one term. The student received a failing grade in all components of the senior practicum course. The student was required to repeat all components of the senior practicum course at her own expense after return from suspension and her graduation was delayed. Upon return from suspension the student was asked to provide a 30-minute in-service to newly admitted nursing students on HIPAA requirements. Without direction from the nursing education program, the student wrote a letter of apology to the clinical agency's Director of Education and Human Resources Department. Within 30 days of the incident, the student was informed by Human Resources at the clinical agency that she could return to work in her role as a CNA and did not face any further disciplinary action. This student did graduate and successfully completed the NCLEX-RN.

\section{Conclusion}

Nursing students are subject to HIPAA requirements and have a professional and ethical obligation to maintain patient 
confidentiality. Nursing education programs, nursing faculty, clinical agencies, nursing students, and nursing preceptors all play important roles in compliance with the HIPAA Privacy Rule. Because of the complexity of issues involved in nursing clinical education, HIPAA violations committed by nursing students during the course of their education must be carefully considered. This case study provides an example of how one baccalaureate nursing education program turned what could have been a terminal mistake into a teachable moment.

\section{REFERENCES}

[1] Ives EJ, Millar S. Caring for patients while respecting their privacy: renewing our commitment. Online Journal of Issues in Nursing. 2005; 10(2): 77.

[2] HHS.gov. Summary of the HIPAA Privacy Rule [Internet]. US Department of Health and Human Services; 1996. Available from: http://www.hhs.gov/hipaa/for-professionals/pri vacy/laws-regulations/index.html

[3] American Nurse. As the web turns. 2005 May/Jun; 37(3): 11.

[4] Federal Register [Internet]. [A rule by the Health and Human Services Department]. Modifications to the HIPAA privacy, security, enforcement, and breach notification rules under the health information technology for economic and clinical health act and the genetic information nondiscrimination act; other modifications to the HIPAA rules. The Daily Journal of the United States Government. 2013.

[5] Bergren M. Information technology, HIPAA and FERPA revisited. Journal of School Nursing. 2004 Apr; 20(2): 107-12. PMid:15040763 http://dx.doi.org/10.1177/10598405040200020901

[6] Gallop Poll [Internet]. Honesty and ethics in professions; 2015. Available from: http://www.gallup.com/poll/1654/honesty -ethics-professions.aspx

[7] Braunack-Mayer A, Mulligan E. Sharing patient information between professionals: confidentiality and ethics. Medical Journal of Australia. 2003 Mar 17; 178(6): 277-9.

[8] Durning M. Consequences of violating the HIPAA privacy rule. Nursing Link Online. 2011. 